\title{
Overcoming obstacles to progression
}

TRF2
depletion was
associated
with reduced
replication
speed and
increased
replication-fork
stalling at
pericentro-
meres

\section{g}

Repeat-rich genomic regions such as centromeres, pericentromeres, telomeres and generally heterochromatin can impede replication and challenge genome integrity. The mechanisms responsible for surpassing such replication impediments are poorly understood. Two papers in Molecular Cell now reveal new factors that support replication at telomeres and at pericentromeric heterochromatin.

Telomeres are protected by the shelterin complex. The shelterin protein telomeric repeat-binding factor 1 (TRF1) promotes telomere replication, likely by recruiting the helicase BLM to resolve DNA structures known as G-quadruplexes, which form at the G-rich telomeric repeats. Another shelterin protein, TRF2, facilitates telomere replication by sensing topological constraints, but its full role in replication is unclear.

Li et al. observed in human cell lines that TRF2 co-localizes at telomeres with the spindle-assembly checkpoint protein BUB3; this co-localization increased considerably during $S$ phase and was mediated by direct TRF2-BUB3 interactions. Fluorescence in situ hybridization experiments in BUB3-depleted cells revealed increased telomere loss and multiple telomeric signals (MTS), which were indicative of telomere replication stress and were detected mostly on the G-rich strand.

BUB3 binds the kinase BUB1, and the heterodimer interacted

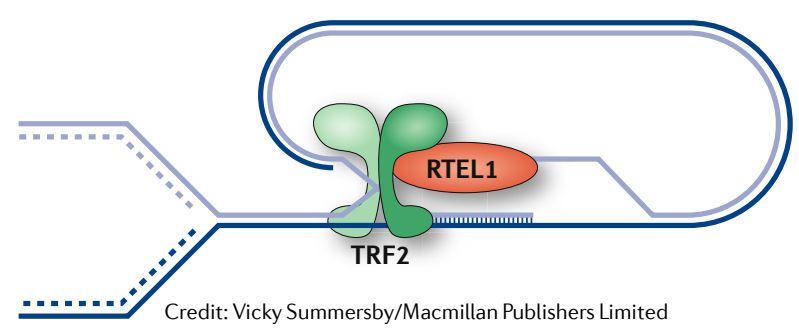

with TRF2 at telomeres. BUB1 depletion or its inhibition during $S$ phase, as well as BLM inhibition, also increased MTS and telomere loss. BUB1 was required for the TRF1-BLM interaction and for maintenance of telomere integrity by phosphorylating TRF1 on Ser296. Thus, the BUB3-BUB1 heterodimer facilitates telomere replication by promoting TRF1 recruitment of BLM, which can resolve telomeric G-quadruplexes.

Mendez-Bermudez et al. explored whether shelterin proteins function in hard-to-replicate regions outside telomeres. TRF2 depletion (but not TRF1 depletion) in human cell lines increased co-localization of pericentromeric repeats with the DNA damage response protein 53BP1, which was visualised by the formation of 'pericentromere dysfunction-induced foci' (PIFs).

TRF2 association with pericentromeric chromatin was validated with various methods and found to be strong, but weaker than its association with telomeres. Importantly, downregulation of the levels of histone H3 Lys9 trimethylation (H3K9me3), which is a modification required for constitutive heterochromatin, promoted TRF2 dissociation from pericentromeres but not from telomeres. However, the dissociation of TRF2 owing to heterochromatin disruption did not trigger pericentromeric damage, suggesting that TRF2 protects pericentromeres from heterochromatin-related DNA damage.

PIF numbers and TRF2 association with pericentromeres increased during $\mathrm{S}$ phase, which was exacerbated by the induction of replication stress, for example, by depletion of replication-associated factors, such as regulator of telomere elongation helicase 1 (RTEL1).
Furthermore, TRF2 depletion was associated with reduced replication speed and increased replication-fork stalling at pericentromeres.

Consistent with the data above, binding of the helicase RTEL1 at pericentromeres was dependent on TRF2. Upon RTEL1 depletion, pericentromeric fork speed decreased and fork stalling and DNA damage increased. Stabilization of G-quadruplexes with bisquinolinium G4 ligands also promoted fork stalling and TRF2 recruitment to pericentromeres, and TRF 2 or RTEL1 depletion increased the levels of G-quadruplexes. Thus, TRF2 and RTEL1 promote replication-fork progression at pericentromeric heterochromatin by suppressing the formation of G-quadruplexes.

Finally, other H3K9me3-enriched chromosomal regions showed DNA damage upon TRF2 depletion, indicating that TRF2 is required for the protection of heterochromatin beyond pericentromeres.

In summary, a uniform mechanism of heterochromatin replication emerges, in which chromatin-conformation-dependent proteins such as TRF2 function at hard-to-replicate DNA to recruit kinases and helicases in order to support fork progression. How these proteins sense replication stress remains to be elucidated.

Eytan Zlotorynski

ORIGINAL ARTICLES Li, F. et al. The BUB3-BUB1 complex promotes telomere DNA replication. Mol. Cell 70, 395-407.e4 (2018) | Mendez-Bermudez, A. et al. Genome-wide control of heterochromatin replication by the telomere capping protein TRF2. Mol. Cell 70, 449-461.e5 (2018) FURTHER READING Lazzerini-Denchi, E. \& Sfeir, A. Stop pulling my strings - what telomeres taught us about the DNA damage response. Nat. Rev. Mol. Cell Biol. 17, 364-378 (2016)| Hänsel-Hertsch, R. et al. DNA G-quadruplexes in the human genome: detection, functions and therapeutic potential. Nat. Rev. Mol. Cell Biol. 18, 279-284 (2017) 